\title{
Evaluation of a space-observed electric field structure for the ability to destabilize inhomogeneous energy-density-driven waves
}

\author{
I. V. Golovchanskaya, B. V. Kozelov, I. V. Mingalev, M. N. Melnik, and A. A. Lubchich \\ Polar Geophysical Institute, Apatity, Russia \\ Correspondence to: I. V. Golovchanskaya (golovchanskaya@pgia.ru) \\ Received: 18 August 2013 - Revised: 17 November 2013 - Accepted: 21 November 2013 - Published: 10 January 2014
}

\begin{abstract}
We examine the effectiveness of nonuniform, quasistatic, transverse electric fields that are often observed in the auroral region in destabilization of inhomogeneous energy-density-driven (IEDD) waves. Specifically, the IEDD dispersion relation of Ganguli et al. (1985a, b) is evaluated for an electric field structure observed by the FAST satellite in the auroral ionosphere at $1000 \mathrm{~km}$ altitude. The background field-aligned current, plasma density and ion composition are derived from FAST observations. Other input parameters adopted in the calculations are varied in pertinent ranges. Unstable solutions are obtained that indicate a variety of frequencies and perpendicular wavelengths. These can manifest as a broadband spectrum of IEDD waves.
\end{abstract}

Keywords. Ionosphere (plasma waves and instabilities)

\section{Introduction}

By now it has been demonstrated in numerous studies that the broadband extremely low frequency (BBELF) waves that are often detected in the auroral ionosphere, as reported by Bonnell et al. (1996), Ergun et al. (1998), Kintner et al. (2000) and others, can hardly be explained in terms of the current-driven electrostatic ion-cyclotron instability (CDEICI) (Drummond and Rosenbluth, 1962; Kindel and Kennel, 1971). The reasons are too low observed values of the parallel drift of electrons, especially at lower $(\leq 1000 \mathrm{~km})$ altitudes, the lack of spectrum ordering by ion gyrofrequencies, the occurrence of intense ion heating in association with BBELF waves, which must lead to CDEICI quenching, etc.

A plausible alternative to the CDEICI is the mechanism proposed by Ganguli et al. (1985a, b). It is based on the observational fact of the presence of nonuniform, quasistatic, perpendicular electric fields during BBELF events (e.g.,
Mozer et al., 1977; Basu et al., 1988; Kintner et al., 1996; Hamrin et al., 2001). The theoretical framework was initially developed by Ganguli et al. (1985a, b) and Ganguli and Palmadesso (1988) for a single layer of transverse, localized electric field (sheared $\boldsymbol{E} \times \boldsymbol{B}$ plasma flow) surrounded by zero electric field regions. It was shown that in such a configuration the dispersive properties of electrostatic waves change dramatically. Due to the Doppler shift of the wave frequency in the plasma reference frame, the energy density $\varepsilon$ of the waves in the region with localized electric field may become negative, while it keeps positive in the surrounding regions. When the regions are coupled by a nonlocal wave packet, a flow of energy from the region of negative $\varepsilon$ to that of positive $\varepsilon$ can enable the mode to grow, giving rise to the instability, which is referred to as the inhomogeneous energydensity-driven (IEDD) instability. Furthermore, in the theoretical studies of Ganguli and Palmadesso (1988), Ganguli et al. (1994), and Gavrishchaka et al. (1996), it was shown that by changing the resonance properties of the system a combination of transverse localized electric fields and fieldaligned currents results in a lower excitation threshold current for CDEIC waves than inferred from the classical theory of the CDEIC instability. This theoretical prediction was validated in a number of laboratory experiments (Koepke and Amatucci, 1992; Amatucci et al., 1994; Koepke et al., 1994). It was also corroborated experimentally that broadband waves in the ion-cyclotron frequency range can be driven solely by a transverse, localized electric field, without the dissipation of a field-aligned current (Amatucci et al., 1998).

Reynolds and Ganguli (1998) generalized the single-layer theory to allow for multiple shear flows. They demonstrated that in a configuration with two flow layers the development of the IEDD instability is facilitated, especially when 
the flow velocities are oppositely directed in adjacent layers because in this case one of the layers can act as an energy sink, thereby provoking destabilization. Reynolds and Ganguli (1998) pointed out that the generalized theory may have implications for the understanding of the auroral ionosphere, where such spatial structures in the transverse electric field often occur.

In this study we verify their prediction by evaluating the IEDD dispersion relation for a structured electric field observed by the FAST satellite in the auroral region. Earlier, the possibility that the nonuniform, quasistatic, transverse electric fields may be important for the generation of broadband waves in the auroral ionosphere was examined by Hamrin et al. (2001) by using Freja observations. Here we expand on the treatment of Hamrin et al. (2001) and provide a more quantitative analysis to confirm the effectiveness of the proposed mechanism.

In the following section the equilibrium configuration, which is adopted from FAST observations, is described. In Sect. 3 we present unstable solutions of the IEDD dispersion relation and demonstrate that for a handful of input parameters they indicate a variety of frequencies and perpendicular wavelengths. The results are summarized and discussed in Sect. 4.

\section{Setting the equilibrium configuration}

We treat an event of structured electric fields observed by the FAST satellite in a crossing of the morning auroral zone on 25 April 1998, UT =00:43:00-00:44:00 (Fig. 1) at $1000 \mathrm{~km}$ altitude. The part of the FAST orbit corresponding to the time interval of interest is marked in bold in Fig. 1a. The north-south electric field $E_{\mathrm{NS}}$, as well as the perturbation of the east-west magnetic field $B_{\mathrm{EW}}$, measured during the event, are shown in Fig. 1b. The sample rate of the electric field measurements is $512 \mathrm{~s}^{-1}$. Under the assumption that the satellite is sampling spatial, rather than temporal variations, whose validity is discussed further in Sect. 4, this corresponds to $14 \mathrm{~m}$ spatial resolution, the FAST velocity being $7.5 \mathrm{~km} \mathrm{~s}^{-1}$. The frequencies below $2 \mathrm{~Hz}$ (scales $>3.5 \mathrm{~km}$ ) are filtered out from the data to avoid contamination from residual spin harmonics (spin period of FAST is $5 \mathrm{~s}$ ). The frequency range of magnetic observations is restricted by $8 \mathrm{~Hz}$ from above because of low-pass filtering of fluxgate magnetometer data on FAST.

Figure 1c plots a wavelet power vs. scale (note the log$\log$ coordinates) for the observed $E_{\mathrm{NS}}$ field, the Daubechies wavelet of the fifth order applied (Daubechies, 1988). It is seen that the power-law dependence (indicated by the solid line) with the slope of $2.2 \pm 0.3$ extends up to frequency of $32 \mathrm{~Hz}(224 \mathrm{~m})$ and then is violated at higher frequencies. Previously, the presence of two types of oscillations in the BBELF signals with a transition region in the frequency (a)

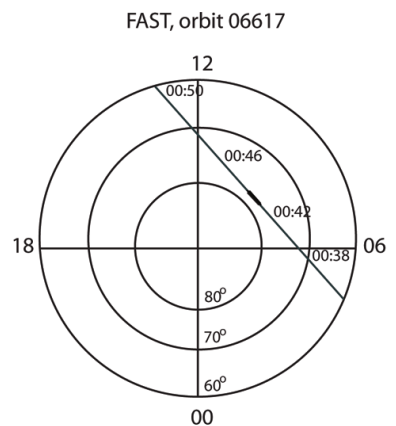

(b)

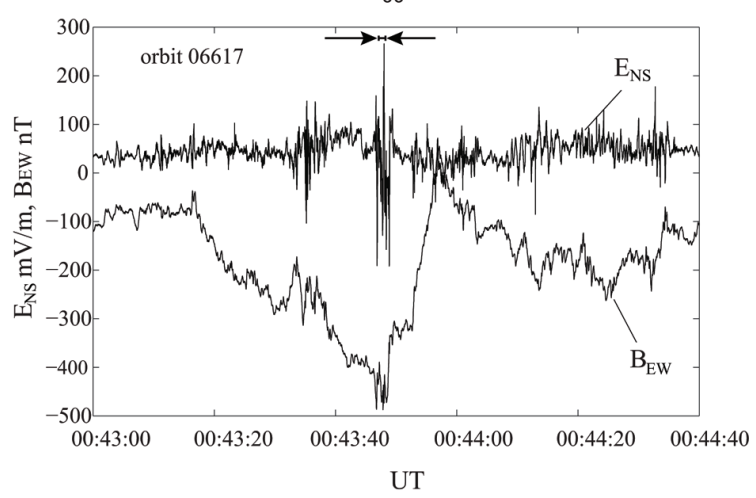

(c)

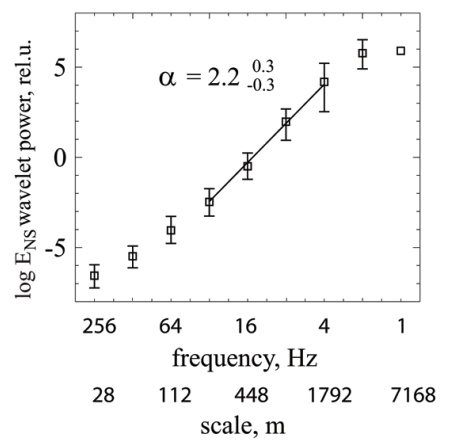

Fig. 1. (a) FAST crossing of the morning auroral zone at $1000 \mathrm{~km}$ altitude on 25 April 1998 (orbit 06617). The time interval of interest is marked in bold; (b) FAST observations of the structured electric fields at UT =00:43:00-00:44:40. Shown are the north-south electric field $E_{\mathrm{NS}}$ measured with $512 \mathrm{~s}^{-1}$ sample rate and the perturbation of the east-west magnetic field $B_{\mathrm{EW}}$. The frequency range of magnetic observations is restricted by $8 \mathrm{~Hz}$ from above. A horizontal bar with arrows indicates the position of an "isolated" electric field structure selected for the analysis; (c) plot of a wavelet power vs. scale in the log-log coordinates for the observed $E_{\mathrm{NS}}$ field, constructed with the Daubechies wavelet of the fifth order applied. The power-law dependence characterized by the slope of $2.2 \pm 0.3$ is indicated by the solid line.

domain at $30-60 \mathrm{~Hz}$ has been reported, for example, by Heppner et al. (1993).

In the present study we adhere to the viewpoint of Kintner et al. (1996), Chang (2001), Tam et al. (2005) and others that the electric fields characterized by the power-law dependence of power vs. scale are predominantly quasistatic and result from some kind of cascading process in the plane transverse 
to the background magnetic field (Chang et al., 2004). In this regard, any structured electric fields at scales $>224 \mathrm{~m}$ in the analyzed data series can be selected to test for the ability to destabilize IEDD waves. At the same time, a nonlocal analysis that will be performed in Sect. 3 to evaluate the effectiveness of the mechanism is formally applicable either to the entire time sequence or over a period where the eigenmodes can be considered to be evanescent at the boundaries. Since it is known that the BBELF emissions are observed as transversely limited bursts of a spatial extent from hundreds of meters to tens of kilometers (e.g., Fig. 2 of Bonnell et al., 1996, Fig. 1 of Ergun et al., 1998, etc.), we inferred the second possibility and searched for "isolated" electric field structures in our data series.

A structure of this type was encountered in the time interval marked by a horizontal bar with arrows in Fig. 1b. As seen from simultaneous magnetic observations, it is embedded in the downward Region 1 field-aligned current. A closeup of the structure is presented in Fig. 2 (top panel), where the dashed lines mark its borders. The bottom middle and right panels in this figure show the same structure being lowpass filtered with a cutoff scale of $448 \mathrm{~m}$ and approximated by a piecewise constant function. A resulting multilayer configuration is examined from the viewpoint of the IEDD theory, as will be described in Sect. 3 .

From FAST magnetic observations the density of the background field-aligned current was estimated as $\sim 30 \times$ $10^{-6} \mathrm{~A} \mathrm{~m}^{-2}$, which for the measured plasma density of $3 \times$ $10^{3} \mathrm{~cm}^{-3}$ corresponded to the parallel drift velocity of electrons equal to $62 \mathrm{~km} \mathrm{~s}^{-1}$.

The measurements of ion composition by the TEAMS instrument on FAST indicated the presence of three ion species, $\mathrm{O}^{+}, \mathrm{He}^{+}$and $\mathrm{H}^{+}$, in the considered time interval. Accordingly, in our calculations we tried three values of the ion-to-electron mass ratio $m_{\mathrm{i}} / m_{\mathrm{e}}$.

To evaluate the IEDD dispersion relation, one also needs the value of temperature ratio $\tau=\frac{T_{\mathrm{i}}}{T_{\mathrm{e}}}$, where $T_{\mathrm{i}}$ and $T_{\mathrm{e}}$ are the ion and electron temperatures. Unfortunately, the lowest energies measured by the electrostatic analyzer (ESA) on FAST were $3 \mathrm{eV}$ for ions and $4 \mathrm{eV}$ for electrons; therefore in our treatment $T_{\mathrm{i}}$ and $T_{\mathrm{e}}$ were free parameters. Using their values observed by other spacecraft in the events of structured electric fields at similar altitudes (e.g., Basu et al., 1988), $T_{\mathrm{i}}$ was varied in the range $0.1-0.6 \mathrm{eV}$ and $\tau$ from 0.1 to 2 . In setting large values of $\tau$, we suppose that $T_{\mathrm{i}}$ is elevated above the background level due to IEDD instability developing in a steady-state structure crossed by FAST.

\section{Evaluation of the IEDD dispersion relation}

We evaluate the IEDD dispersion relation of Ganguli et al. (1985a, b) for the $\boldsymbol{E}$ field configuration shown in Fig. 2, bottom right panel, and for assumptions made plausible from space observations described in the previous section.
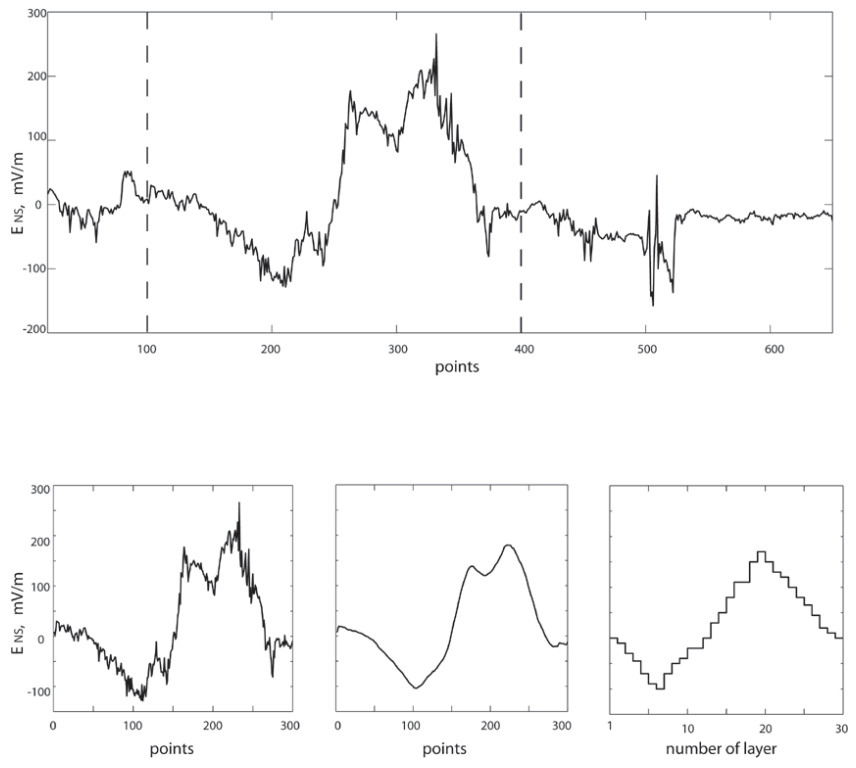

Fig. 2. (top) An "isolated" electric field structure selected to be evaluated by the IEDD theory. The dashed lines mark the borders of the structure; (bottom) the same structure being smoothed and approximated by a piecewise constant function.

Following Ganguli et al. (1985a, b), Gavrishchaka et al. (1996), Reynolds and Ganguli (1998), we consider the dispersion relation for the electrostatic ion-cyclotron (EIC) mode in the nonuniform plasma, where non-uniformities in the $x$ direction are included by suggesting small values of the wave vector component $k_{x}$, so that the substitution $k_{x} \rightarrow-i \partial / \partial x$ can be made (the background configuration is assumed uniform in the $y$ and $z$ directions). The plasma response then results in the second-order differential equation for the electrostatic potential $\phi$. When the plasma parameters are piecewise constant, this (eigenvalue) equation can be written in each region as

$\left(\frac{\partial^{2}}{\partial \xi^{2}}+\kappa^{2}\right) \phi(\xi)=0$,

where $\xi=\frac{x}{\rho_{i}}, \rho_{i}$ the ion Larmor radius,

$$
\begin{aligned}
& \kappa^{2}(\xi)= \\
& -2 \frac{1+\tau+\sum_{n} \Gamma_{n}(b)\left(\frac{\omega_{1}}{\left|k_{\|}\right| \mid V_{\mathrm{ti}}}\right) \mathrm{Z}\left(\frac{\omega_{1}-n \Omega_{i}}{\left|k_{\mid}\right| \mid V_{\mathrm{ti}}}\right)+\tau\left(\frac{\omega_{1}-k_{\|} \mid V_{d}}{\left|k_{\mid}\right| \mid V_{\mathrm{te}}}\right) \mathrm{Z}\left(\frac{\omega_{1}-k_{\mid} \mid V_{\mathrm{d}}}{\left|k_{k}\right| \mid V_{\mathrm{te}}}\right)}{\sum_{n} \Gamma_{n}^{\prime}(b)\left(\frac{\omega_{1}}{\left|k_{\|}\right| V_{\mathrm{ti}}}\right) Z\left(\frac{\omega_{1}-n \Omega_{i}}{\left|k_{k}\right| \mid V_{\mathrm{ti}}}\right)}
\end{aligned}
$$

and the general solution for $\phi$ in each region is a superposition of two exponentials: $\sim \exp (+i \kappa x)$ and $\sim \exp (-i \kappa x)$. In Eq. (2), $k^{2} \lambda_{i}^{2}<<1$ is assumed, where $\lambda_{i}$ is the Debye length for ions, $\Gamma_{n}(b)=I_{n}(b) \exp (-b)$, where $b=\frac{\left(k_{y} \rho_{i}\right)^{2}}{2}, I_{n}$ are the modified Bessel functions, $n$ being integer, $\Gamma_{n}^{\prime}=\frac{\partial}{\partial b} \Gamma_{n}$, $\omega_{1}=\omega-k_{y} V_{E}, \omega=\omega_{r}+i \gamma, V_{\mathrm{d}}$ is the parallel electron drift velocity (the external magnetic field $B_{0}$ is in the $z$ direction), 
$V_{\mathrm{ti}}$ and $V_{\mathrm{te}}$ are the thermal velocities of ions and electrons, respectively, and $Z$ is the plasma dispersion function. The usual large (small) argument expansion is applied for the ion (electron) $Z$ function (e.g., Drummond and Rosenbluth, 1962). In the present study we retain $n=0, \pm 1$ harmonics for ions and $n=0$ harmonic for electrons, and use that $\Gamma_{0}\left(b_{\mathrm{e}}\right)=1$ for $b_{\mathrm{e}} \equiv \frac{\left(k_{y} \rho_{\mathrm{e}}\right)^{2}}{2} \ll 1$.

The nonlocal dispersion relation is obtained for the piecewise constant flow profile by matching both $\phi$ and $\partial \phi / \partial x$ across the boundaries between the layers and assuming that the solution fades in the outermost regions. In the case of $N_{\mathrm{L}}$ layers we have

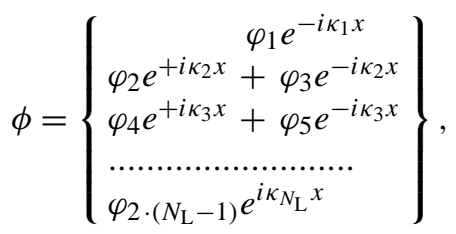

where $\operatorname{Im}\left(\kappa_{1}\right)>0, \operatorname{Im}\left(\kappa_{N_{\mathrm{L}}}\right)>0$. The two matching conditions (continuity of $\phi$ and of $\frac{\partial \phi}{\partial x}$ across each boundary) applied at $N_{\mathrm{L}}-1$ boundaries result in a set of $2 \cdot\left(N_{\mathrm{L}}-1\right)$ equations for amplitudes $\varphi_{1}, \varphi_{2}, \ldots, \varphi_{2 \cdot\left(N_{\mathrm{L}}-1\right)}$ of the potential, which can be written as a matrix equation:

$\mathbf{M} \cdot \varphi=0$.

Setting the determinant of the matrix $\mathbf{M}$ to zero gives a nonlocal dispersion relation:

$\operatorname{det} \mathbf{M}=0$.

A shooting code was used to find roots of the nonlocal dispersion relation in Eq. (5), which has multiple eigenstates in the presence of velocity shears for each set of parameters (for discussion see Reynolds and Ganguli, 1998).

Since from our analysis it appeared that for $\mathrm{O}^{+}$ions IEDD waves are destabilized more readily, that is, for a wider range of the input parameters, we present the results for this particular species $\left(\mu \equiv m_{\mathrm{i}} / m_{\mathrm{e}}=29392\right)$. For definiteness $T_{\mathrm{i}}=$ $0.5 \mathrm{eV}$ is set, which corresponds to $\rho_{\mathrm{i}}=10 \mathrm{~m}$, given the geomagnetic field $B_{0}$ at $1000 \mathrm{~km}$ altitude is about $40000 \mathrm{nT}$. The ion thermal velocity is $V_{\mathrm{ti}}=2.4 \mathrm{~km} \mathrm{~s}^{-1}$, and the electron thermal velocity $V_{\mathrm{te}}=(\mu / \tau)^{1 / 2} \cdot V_{\mathrm{ti}}$.

Figure 3 plots the growth rate $\gamma$ normalized by the $\mathrm{O}^{+}$gyrofrequency ( $\gamma>0$ indicating destabilization) as a function of $b$ for four different real frequencies $\omega_{\mathrm{r}}$ of the IEDD instability that develops in a nonuniform electric field configuration under study. The dashed line refers to $\omega_{\mathrm{r}}=0.94$, thick solid line to $\omega_{\mathrm{r}}=0.96$, dashed-dotted line to $\omega_{\mathrm{r}}=0.98$, and thin solid line to $\omega_{\mathrm{r}}=0.99$, where $\omega_{\mathrm{r}}$ is the real frequency normalized by $\Omega_{\mathrm{i}}$. Other parameters are $\tau=0.5, V_{\mathrm{d}} / V_{\mathrm{te}}=0.1$ and $u \equiv k_{\|} / k_{\perp}=0.1$. The dotted line in Fig. 3 indicates a CDEIC solution that would appear (at $\omega_{\mathrm{r}} / \Omega_{\mathrm{i}}=1.245$ ) if the nonuniform electric fields were removed, $V_{\mathrm{d}} / V_{\mathrm{te}}$ increased to 0.2 and $u$ taken as 0.05 .

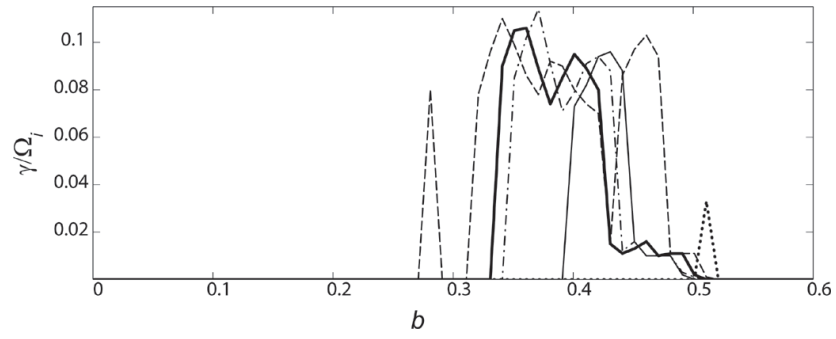

Fig. 3. Plots of the growth rate normalized by the ion gyrofrequency vs. $b \equiv \frac{\left(k_{y} \rho_{i}\right)^{2}}{2}$ for the IEDD instability that develops in a nonuniform electric field configuration shown in Fig. 2, bottom right panel. The dashed line refers to $\omega_{\mathrm{r}}=0.94$, thick solid line to $\omega_{\mathrm{r}}=0.96$, dashed-dotted line to $\omega_{\mathrm{r}}=0.98$, and thin solid line to $\omega_{\mathrm{r}}=0.99$, where $\omega_{\mathrm{r}}$ is the real frequency normalized by $\Omega_{\mathrm{i}}$. Other parameters are $\tau=0.5, V_{\mathrm{d}} / V_{\mathrm{te}}=0.1$ and $u \equiv k_{\|} / k_{\perp}=0.1$. The dotted line indicates a CDEIC solution that would appear (at $\omega_{\mathrm{r}} / \Omega_{i}=1.245$ ) if the nonuniform electric fields were removed, $V_{\mathrm{d}} / V_{\mathrm{te}}$ increased to 0.2 and $u$ taken as 0.05 . The CDEIC solution degenerates for $\tau>0.5$.

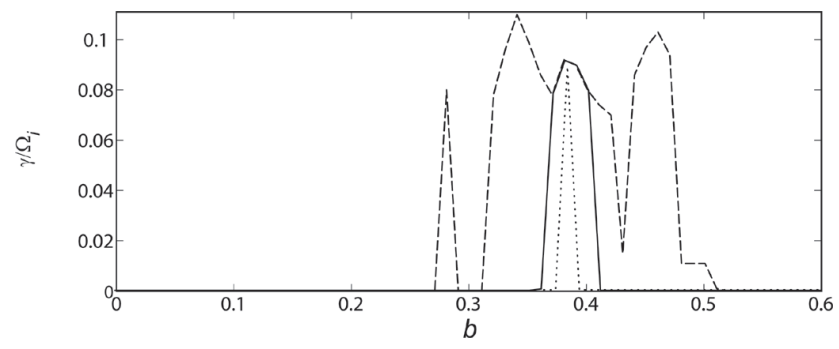

Fig. 4. Behavior of the IEDD solutions shown in Fig. 3 for $\omega_{\mathrm{r}}=$ 0.94 with varying temperature ratio $\tau$ : the dashed line refers to $\tau=$ 0.5 , solid line to $\tau=1$, and dotted line to $\tau=1.2$.

In the runs illustrated in Fig. 3 and further in Figs. 4 and 5, we used a stepwise approximation of the steady-state structure by 30 layers of constant width (shown in the bottom right panel in Fig. 2). We also tried other approximations with different number of layers ( $N_{\mathrm{L}}$ ranging from 30 to 3 ) of both constant and variable widths. It was verified that unstable solutions are persistent to changes in $N_{\mathrm{L}}$, slightly shifting to smaller $b$ values for coarser approximations of $E(x)$ in the structure.

Generally, the solutions for IEDD instability depend on the temperature ratio $\tau$, getting scarcer with increasing $\tau$. However, this dependence is much weaker than that for CDEIC instability. For instance, the CDEIC root shown in Fig. 3 by the dotted line degenerates already at $\tau=0.6$, while some of the IEDD roots typically persist at $\tau>1$. This can be seen in Fig. 4, where the evolution of the IEDD solutions obtained for $\omega_{\mathrm{r}}=0.94$ (dashed line in Fig. 3) with increasing $\tau$ is presented.

Since the ratio $u \equiv k_{\|} / k_{\perp}$ is not available from FAST observations, in our treatment it was a free parameter. We found that there are IEDD solutions for $u$ ranging from 0.05 to 


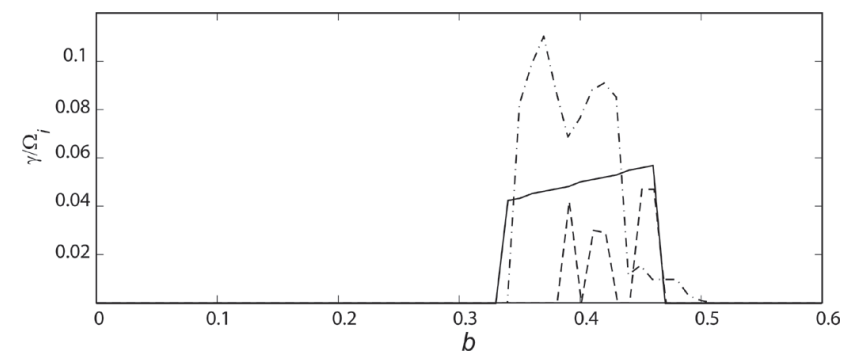

Fig. 5. Behavior of the IEDD solutions shown in Fig. 3 for $\omega_{\mathrm{r}}=$ 0.98 with varying $u$ : the dashed line refers to $u=0.05$, dasheddotted line to $u=0.1$, and solid line to $u=0.2$.

$\sim 0.2$ (Fig. 5) if $\omega_{\mathrm{r}}=0.98$ and all other parameters are such as those adopted in the case shown in Fig. 3. This range of $u$ is consistent with the range $0<u<0.22$ determined by Bonnell et al. (1996) from interferometric observations of BBELF phase velocities with AMICIST sounding rocket instrumentation.

\section{Discussion}

Accurate knowledge of the generation mechanism of the broadband electrostatic ELF waves in the auroral topside ionosphere is important, taking into account high correlation between this emission and ion transverse heating (e.g., André et al., 1998; Lund et al., 2000). In the previous sections we demonstrated that the nonuniform electric fields (multiple shear layers) that are actually observed in the auroral region can destabilize the broadband electrostatic waves via the mechanism of IEDD instability proposed by Ganguli et al. (1985a, b) and generalized by Ganguli and Palmadesso (1988), Gavrishchaka et al. (1996), Reynolds and Ganguli (1998) and others.

One problem concerning the applicability of the theory of inhomogeneous energy-density-driven instability (IEDDI) is that the nonuniform electric fields in the auroral ionosphere considered to be a driver of IEDD instability usually have a magnetic counterpart (e.g., Mishin and Förster, 1995; Golovchanskaya et al., 2011), and thus a Poynting flux is present in the background configuration. Throughout this paper it was assumed that the magnetic counterpart is produced by quasistatic field-aligned currents that are closed to the ionosphere (Vickery et al., 1986; Golovchanskaya et al., 2013), so that the Poynting flux input to the system dissipates in the conductive ionosphere and does not invalidate a quasi-static energy density consideration on which the IEDDI theory is based. Furthermore, generalization of the IEDDI theory to the electromagnetic regime was accomplished by Peñano and Ganguli (1999, 2002). A class of unstable electromagnetic ion cyclotron (EMIC) eigenmodes were found. Tejero et al. (2011) reported on laboratory observations of EMIC waves generated by a localized transverse direct-current electric field. Peñano and Ganguli (1999, 2002) argue that electromagnetic IEDDI could account for the Poynting flux that originates from the ionosphere, being observed by satellites at a higher altitude.

As already mentioned, in the present study it is assumed that the frequencies in the satellite frame are Doppler-shifted frequencies, resulting from satellite traversing spatial structures. Clearly, this is true for quasistatic fields. We note that this is also justified for $\mathrm{O}^{+}$IEDD waves, since the FAST velocity of $7.5 \mathrm{~km} \mathrm{~s}^{-1}$ several times exceeds their transverse phase velocity.

While in this brief report the results have been presented for a single event of the structured electric field observed by FAST in the auroral region (shown in Fig. 2), we tried several other structures of this type and verified their effectiveness in destabilizing IEDD waves. Generally, a common feature of the unstable IEDD solutions obtained for multilayer configurations is a variety of modes, which differ in frequencies and perpendicular wave numbers and persist even for zero parallel electron drift, as opposed to the solutions for the CDEIC instability (Kindel and Kennel, 1971), which occur for a single $\omega_{\mathrm{r}}$ and a single $b$, and require strong parallel electron drift.

Acknowledgements. Authors acknowledge the NASA National Space Science Data Center and R. C. Elphic, R. E. Ergun, R. J. Strangeway, and C. W. Carlson for FAST data usage. This work was supported by Program 22 of the Presidium of the Russian Academy of Sciences (RAS) "Fundamental problems of the Solar system exploration" and grant 13-01-00063a of RFBR.

Topical Editor K. Hosokawa thanks E. V. Mishin and one anonymous referee for their help in evaluating this paper.

\section{References}

Amatucci, W. E., Koepke, M. E., Carroll III, J. J., and Sheridan, T. E.: Observation of ion-cyclotron turbulence at small values of magnetic-field-aligned current, Geophys. Res. Lett., 21, 15951598, 1994.

Amatucci, W. E., Walker, D. N., Ganguli, G., Duncan, D., Antoniades, J. A., and Bowles, J. H.: Velocity-shear-driven ioncyclotron waves and associated transverse ion heating, J. Geophys. Res., 103, 11711-11724, 1998.

André, M., Norqvist, P., Andersson, L., Eliasson, L., Eriksson, A. I., Blomberg, L., Erlandson, R. E., and Waldemark, J.: Ion energization mechanisms at $1700 \mathrm{~km}$ in the auroral region, J. Geophys. Res., 103, 4199-4222, 1998.

Basu, S., Basu, S., Mackenzie, E., Fougere, P. F., Coley, W. R., Maynard, N. C., Winningham, J. D., Sugiura, M., Hanson, W. B., and Hoegy, W. R.: Simultaneous density and electric field fluctuation spectra associated with velocity shears in the auroral oval, J. Geophys. Res., 93, 115-136, 1988.

Bonnell, J., Kintner, P., Wahlund, J.-E., Lynch, K., and Arnoldy, R.: Interferometric determination of broadband ELF wave phase velocity within a region of transverse auroral ion acceleration, Geophys. Res. Lett., 23, 3297-3300, 1996. 
Chang, T.: Colloid-like behavior and topological phase transitions in space plasmas: Intermittent low frequency turbulence in the auroral zone, Phys. Scripta, T89, 80, doi:10.1238/Physica.Topical.089a00080, 2001.

Chang, T., Tam, S. W. Y., and Wu, C.: Complexity induced anisotropic bimodal intermittent turbulence in space plasmas, Phys. Plasma, 11, 1287-1299, 2004.

Daubechies, I.: Orthonormal bases of compactly supported wavelets, Commun. Pure Appl. Math., 41, 909-996, 1988.

Drummond, W. E. and Rosenbluth, M. N.: Anomalous diffusion arising from microinstabilities in a plasma, Phys. Fluids, 5, 1507-1513, 1962.

Ergun, R. E., Carlson, C. W., McFadden, J. P., Mozer, F. S., Delory, G. T., Peria, W., Chaston, C. C., Temerin, M., Elphic, R., Strangeway, R., Pfaff, R., Cattell, C. A., Klumpar, D., Shelley, E., Peterson, W., Moebius, E., and Kistleret, L.: FAST satellite observations of electric field structures in the auroral zone, Geophys. Res. Lett., 25, 2025-2028, 1998.

Ganguli, G. and Palmadesso, P. J.: Electrostatic ion instabilities in the presence of parallel currents and transverse electric fields, Geophys. Res. Lett., 15, 103-106, 1988.

Ganguli, G., Palmadesso, P., and Lee, Y. C.: A new mechanism for excitation of electrostatic ion cyclotron waves and associated perpendicular ion heating, Geophys. Res. Lett., 12, 643-646, 1985a.

Ganguli, G., Lee, Y. C., and Palmadesso, P.: Electrostatic ioncyclotron instability caused by a nonuniform electric field perpendicular to the external magnetic field, Phys. Fluids, 28, 761763, 1985b.

Ganguli, G., Keskinen, M. J., Romero, H., Heelis, R., Moore, T., and Pollock, C.: Coupling of microprocesses and macroprocesses due to velocity shear: An application to the low-altitude ionosphere, J. Geophys. Res., 99, 8873-8889, 1994.

Gavrishchaka, V., Koepke, M. E., and Ganguli, G.: Dispersive properties of a magnetized plasma with a field-aligned drift and inhomogeneous transverse flow, Phys. Plasmas, 3, 3091-3106, 1996.

Golovchanskaya, I. V., Kozelov, B. V., Mingalev, O. V., Fedorenko, Y. V., and Melnik, M. N.: Magnetic perturbations in the events of broadband ELF turbulence observed by FAST, Geophys. Res. Lett., 38, L17103, doi:10.1029/2011GL049003, 2011.

Golovchanskaya, I. V., Kozelov, B. V., and Despirak, I. V.: Seasonal asymmetry in the broadband ELF electric fields observed by the FAST satellite, J. Atmos. Sol.-Terr. Phys., 99, 111-116, doi:10.1016/j.jastp.2012.12.011, 2013.

Hamrin, M., André, M., Ganguli, G., Gavrishchaka, V. V., Koepke, M. E., Zintl, M. W., Ivchenko, N., Karlsson, T., and Clemmons, J. H.: Inhomogeneous transverse electric fields and wave generation in the auroral region: A statistical study, J. Geophys. Res., 106, A6, 10803-10816, 2001.

Heppner, J. P., Liebrecht, M. C., Maynard, N. C., and Pfaff, R. F.: High-latitude distributions of plasma waves and spatial irregularities from DE2 alternating current electric field observations, J. Geophys. Res., 98, 1629-1652, 1993.
Kindel, J. M. and Kennel, C. F.: Topside current instabilities, J. Geophys. Res., 76, 3055-3078, 1971.

Kintner, P. M., Bonnel, J., Arnoldy, R., Lynch, K., Pollock, C., and Moore, T.: SCIFER - Transverse ion acceleration and plasma waves, Geophys. Res. Lett., 23, 1873-1876, 1996.

Kintner, P. M., Franz, J., Schuck, P., and Klatt, E.: Interferometric coherency determination of wavelength or what are broadband ELF waves?, J. Geophys. Res., 105, 21237-21250, 2000.

Koepke, M. E. and Amatucci, W. E.: Electrostatic ion-cyclotron wave experiments in the WVU Q-machine, IEEE T. Plasma Sci., 20, 631-635, 1992.

Koepke, M. E., Amatucci, W. E., Carroll, J. J., and Sheridan, T. E.: Experimental verification of the inhomogeneous energy-density driven instability, Phys. Rev. Lett., 72, 3355-3358, 1994.

Lund, E. J., Möbius, E., Carlson, C. W., Ergun, R. E., Kistler, L. M., Klecker, B., Klumpar, D. M., McFadden, J. P., Popecki, M. A., Strangeway, R. J., and Tung, Y. K.: Transverse ion acceleration mechanism in the aurora at solar minimum: occurrence distributions, J. Atmos. Sol.-Terr. Phys., 62, 467-475, 2000.

Mishin, E. V. and Förster, M.: "Alfvénic shocks" and low-altitude auroral acceleration, Geophys. Res. Lett., 22, 1745-1748, 1995.

Mozer, F. S., Carlson, C.W., Hudson, M. K., Torbert, R. B., Parady, B., Yatteau, J., and Kelley, M. C.: Observations of paired electrostatic shocks in the polar magnetosphere, Phys. Rev. Lett., 38, 292-295, 1977.

Peñano, J. R. and Ganguli, G.: Ionospheric source for lowfrequency broadband electromagnetic signatures, Phys. Rev. Lett., 83, 1343-1346, 1999.

Peñano, J. R. and Ganguli, G.: Generation of electromagnetic ion cyclotron waves in the ionosphere by localized transverse dc electric fields, J. Geophys. Res., 107, SIA14.1-SIA14.7, doi:10.1029/2001JA000279, 2002.

Reynolds, M. A. and Ganguli, G.: Ion Bernstein waves driven by two transverse flow layers, Phys. Plasmas, 5, 2504-2512, 1998.

Tam, S. W. Y., Chang, T., Kintner, P. M., and Klatt, E.: Intermittency analyses on the SIERRA measurements of the electric field fluctuations in the auroral zone, Geophys. Res. Lett., 32, L05109, doi:10.1029/2004GL021445, 2005.

Tejero, E. M., Amatucci, W. E., Ganguli, G., Cothran, C. D., Crabtree, C., and Thomas Jr., E.: Spontaneous electromagnetic emission from a strongly localized plasma flow, Phys. Rev. Lett., 106, 185001, doi:10.1103/PhysRevLett.106.185001, 2011.

Vickrey, J. F., Livingston, R. C., Walker, N. B., Potemra, T. A., Heelis, R. A., Kelley, M. C., and Rich, F. J.: On the currentvoltage relationship of the magnetospheric generator at intermediate spatial scales, Geophys. Res. Lett., 13, 495-498, 1986. 